\title{
Equipment's Properties Design and Digital Platform
}

\author{
Jiaju Wu*1 ${ }^{*}$ Zhengji Liü ${ }^{2}$,Zheng Cheng ${ }^{2}$,CuiFang Zheng ${ }^{2}$, \\ Xinglin $\mathrm{Zhu}^{2}$, Yongqi $\mathrm{Ma}^{2}$, \\ ${ }^{1}$ China Academy of Engineering Physics, Institute of Computer Application. \\ Mianyang Sichuan, 621999 P.R. China. (PH) +86-131-9807-8524, IEEE member, ID: 41631773, E- \\ mail:wuij@caep.cn. \\ ${ }^{2}$ China Academy of Engineering Physics, Institute of Computer Application. \\ Mianyang Sichuan, 621999 PR. China.
}

\begin{abstract}
Keywords - properties design and analysis, equipment, digital, five properties , platform.
Abstract - Equipment's property includes reliability, maintainability, testability, supportability and safety, which are called five properties. At present, weapons equipments function and performance design is separated from equipments' five properties design. And model design persons' work environment is isolation from five properties persons' work environment. These factors make cooperative database sharing difficulties. With the analysis the digital integrated support experience of Europe and the United States in reliability, maintainability, supportability, testability and safety analysis and design work, this paper puts forward five properties design and analysis digital platform solutions, which is used to solve weapon design, support equipment design, equipment property, design. The functions, architecture and key technologies of the platform are discussed.
\end{abstract}

\section{Introduction}

From 80s years, developed countries make full use of the rapid development of the information technology progress. They continuous improve equipment comprehensive support strategy, reliability, maintainability, supportability, testability and safety design and analysis, which are called "five properties" design and analysis. They have carried out computer aided life cycle support to accelerate the product development process, improve equipment's availability and reduce equipment life cycle cost of whole weapon system [1,2]. The project has taken the transition from traditional "paper" to "electronic business", which is showing the characteristics of system engineering integration, digitalization, information and standardization. The basic idea is "create once, reuse often" in the data source generation. The basic implementation mode is system integration. The basic method is to construct integrated covering product design, support, and service function of concurrent and collaborative engineering environment based on information integration. And it generates integrated weapon system knowledge database, which reflects the basic characteristics of digitization, information, standardization, integration. A number of domestic equipment manufacturing and scientific research units to carry out the "five properties" design work. They have established a variety of analysis software and systems. However, most of the departments are still in the primary stage, whose equipment system's "two skins" problem of function / performance design and the "five properties" design and analysis work's is still not been fundamentally solved. Concrete details are embodied as following.

(1) "Five properties" work lags badly behind the function \& performance design.

(2) For the lack of integration of work environment and professional software for engineering application support, "five properties" design and model design personnel has not high work efficiency, the low work level, too simplified results etc., when they carry out the work of the "five properties" design and analysis.

(3) "Five properties" data flow of information is poor. And it can not achieve the function / performance data between the integrated information environments reasonably and efficiently. So "five properties" design information islands have occurred. 


\section{Five design and analysis of digital platform requirements}

The "five properties" design and analysis digital platform needs to achieve equipment reliability, maintainability, supportability, testability and safety design and analysis, integrated and unified management of basic data and "“five properties"” work management. Specific demand for:

(1) Equipment's reliability design and analysis [7] needs to achieve equipment reliability prediction, reliability allocation, failure mode effect and criticality analysis, reliability analysis, network diagram of reliability analysis, fault tree analysis, event tree analysis, Markov analysis, reliability data evaluation and analysis etc.

(2) Equipment's maintainability design and analysis [6] needs to be achieved. Maintainability design and analysis is one of the major maintenance activities in the process of development, which is based on historical experience and similar product data estimation, calculation of maintainability parameters of new products in the given working conditions, in order to understand the design to meet the maintenance requirements of the degree. The platform mainly realizes maintainability prediction, failure mode and effect analysis and maintainability analysis.

(3) Equipment's testability design and analysis [5] should be implemented. The platform establishes test model for analysis objects, which rely fault relation. Then it also designs on how to carry out maintenance work based on test index and current conditions. And it designs the detail of work process. At the same time of the testability design, it allows users to consider malfunction, testing recommendations, operational recommendations, maintenance equipment list, components reliability index, the cost of testing time, cost of maintenance time and other parameters as the design of system maintenance elements. It can do static analysis and test analysis for system simulation model. Through the analysis report (group of fuzzy report, redundancy report, without detection report, feedback loop report, disconnect feedback loop reports, test quality map, etc.), the platform can evaluate and optimize the system testability, and reduce the life cycle cost of the whole system.

(4) Equipment's supportability design and analysis needs to be achieved. The platform should realize equipments' supportability design and analysis, reliability centered maintenance analysis, using and maintenance task analysis, repair level analysis, cost analysis and forecast of spare parts demand, support integration optimization analysis (risk analysis, balance analysis, sensitivity analysis).

(5) Equipment's safety design and analysis needs to be achieved. The platform should realize equipment safety design and analysis, failure mode and effect analysis, fault tree analysis, event tree analysis, hazard and operability analysis, risk assessment analysis, network availability analysis.

(6) Five properties foundation database management should be realized in digital platform. "Five properties" integrated knowledge management manages general data, engineering data and proprietary data changes, data baseline, product breakdown structure, failure mode and support resources. According to user requirements, it integrates data to generate normal or custom-made support scheme. In order to make the information stored in general formality and reduce the conversion process of data format not currency in different platforms, we establish equipment "five properties" integration knowledge database according to S3000L, MIL-STD-1388-2B,GJB3872,GJB3837 standard.

(7) Digital platform should sustain "five properties" design work management. The concrete work management includes RCM project management, multimedia annotation file management, process management, engineering change management, analysis and comparison of state technology and other professional data management interface. The equipment system involved professional and engineering departments, the large number of personnel. The digital platform combined into an ordered whole and collaborative engineering activities environment, which is not unable to guarantee the performance of largescale projects and the final product (including support) by the traditional management mode of the personnel physical assembly, paper documents etc. Digital platform need to achieve unity process and data.

\section{"Five properties" design and analysis digital platform architecture}

"Five properties" design and analysis digital platform adapts reliability, maintainability, support, testing and safety design and analysis work demand as the starting point. Through the overall planning and design 
integration, we research and develop advanced "five properties" design and analysis of digital platform (including the "five properties" management system, "five properties" and design and analysis system, five basic database) which uses the database as the foundation, management system for the management of centralized control center and the design and analysis of data sharing between systems. Platform architecture is shown in figure 1.

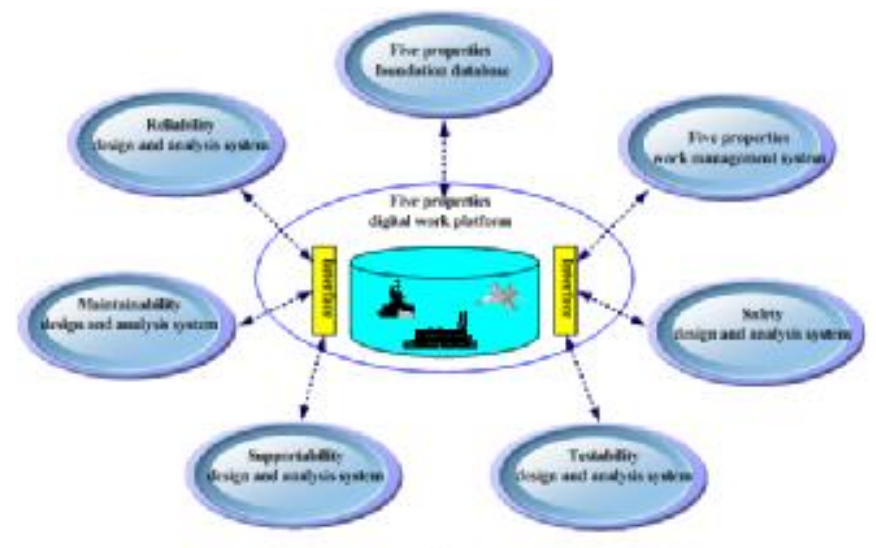

Figure 1. "Five properties" design and analysis digital platform

"Five properties" design and analysis digital platform structure is shown in figure 3. The "five properties" design and analysis of digital platform specific functions include reliability design and analysis system, maintainability design and analysis system, support design and analysis system, test design and analysis, system safety design analysis system, basic database and management system. "Five properties" design and analysis work flow is like figure 4.

\section{Platform implementation}

The digital platform software adapts advanced J2EE multi-layer architecture technology, which is like figure 4. The digital platform implements technology includes component technology, Spring framework technology, Hibernate Data Persistence technology, MiniUI component development technology and support cross domain classification management mode. The platform uses the browser / server (B/S) architecture, which the user interfaces, business logic, system maintenance, maintenance assessment process are logic separation. The information system function and data representation, according to the function is divided into three blocks. The database system adopts the Oralce 10g. Workflow engine is developed by CAEP, which is named SWCCFlowServer. And Jboss Enterprise Edition is used as application server. Run operating system is Linux which is considered with security point of view using. The client accesses server through IE browser. 


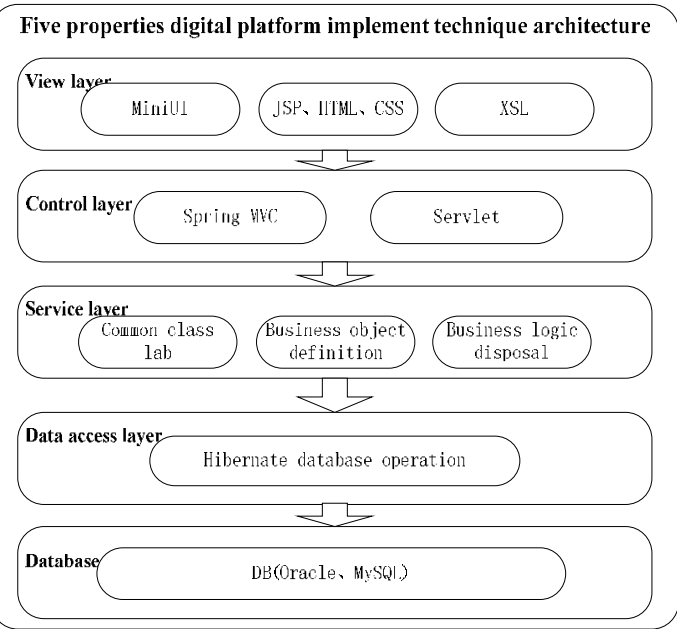

Figure 2. "Five properties" design and analysis digital platform
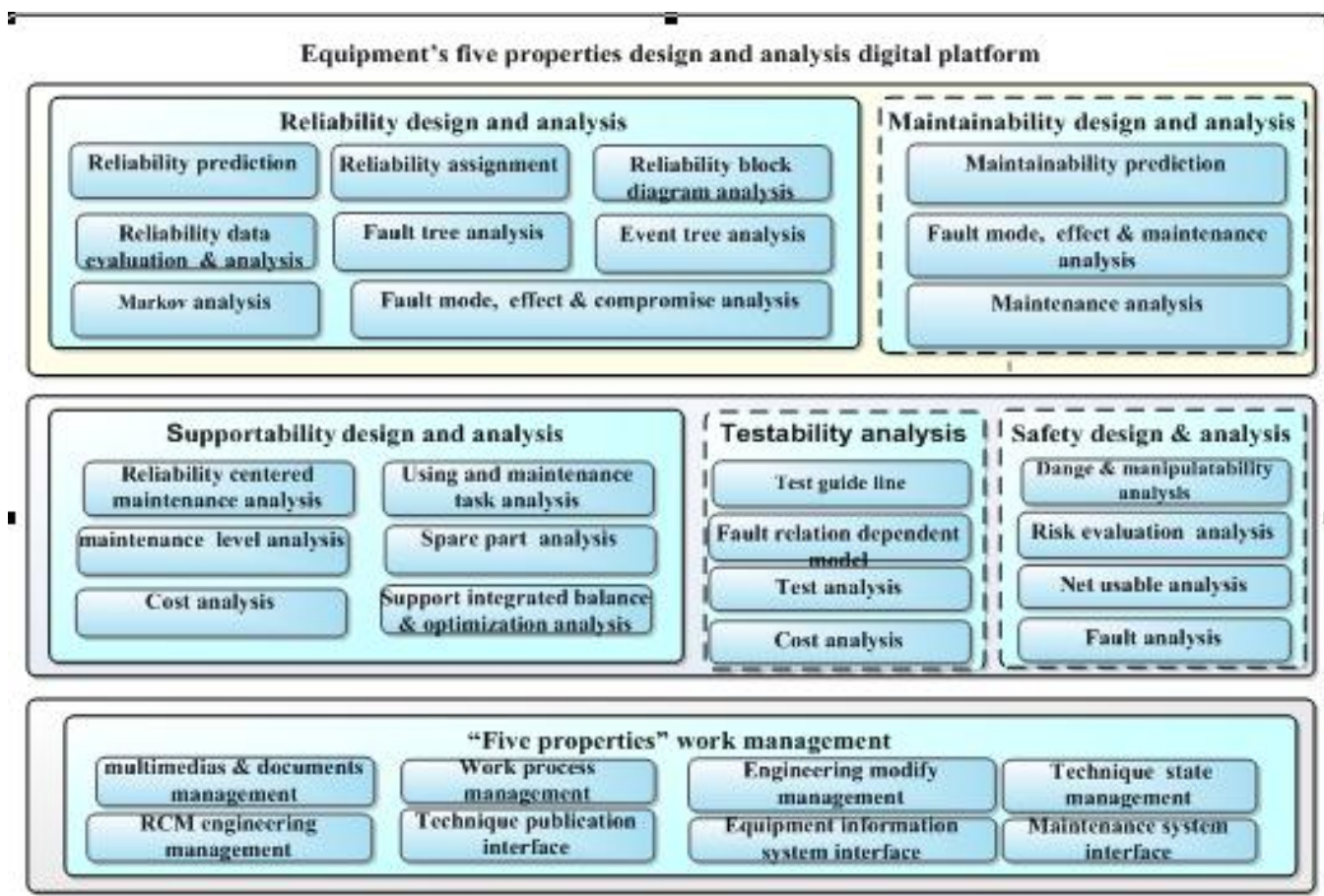

Foundation data management

"Five properties" design and analysis digital platform 


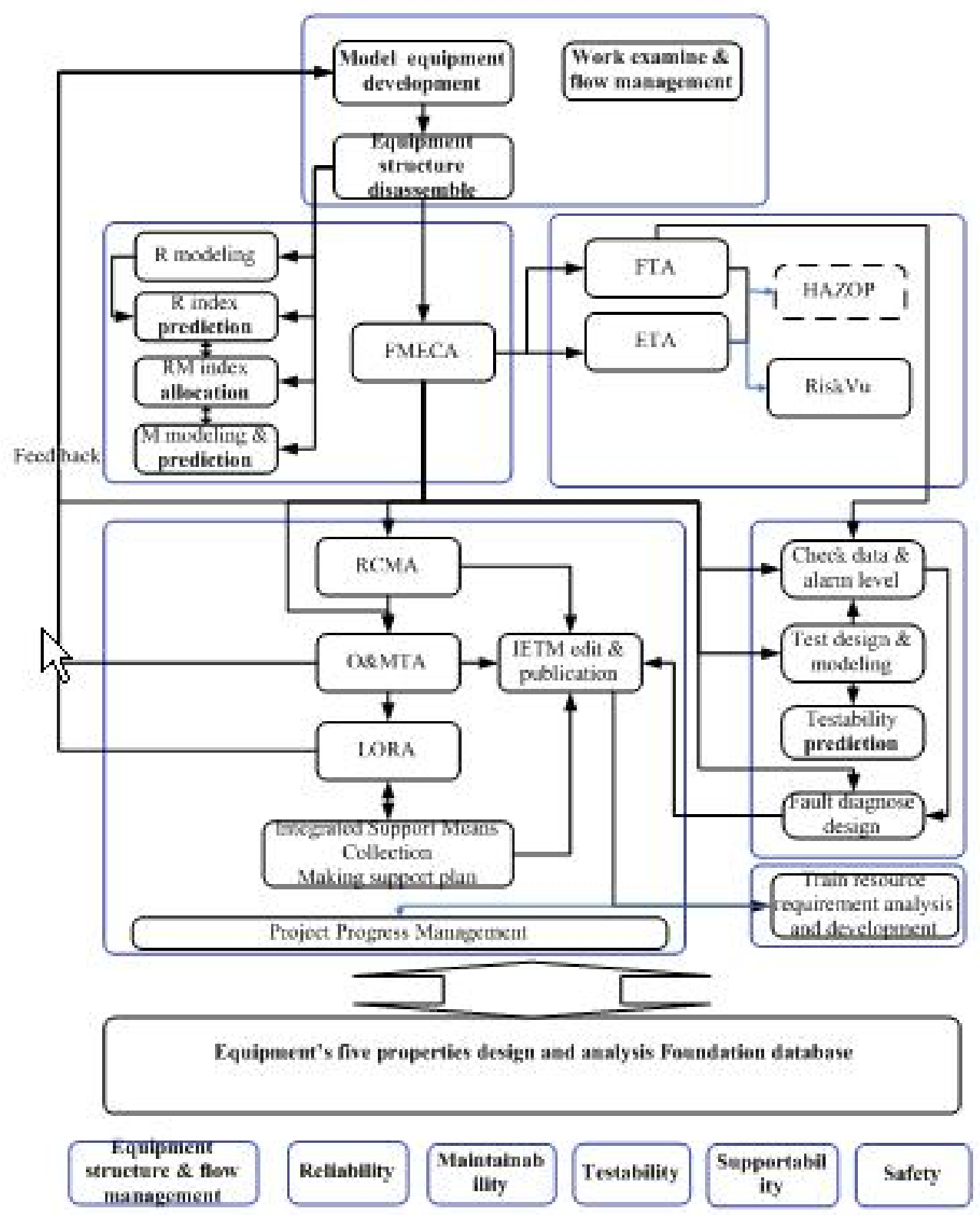

Figure 3. "Five properties" design and analysis work flow 


\section{CONCLUSION}

Through the establishment and implementation of the "five properties" integration platform, it provides a stable, efficient, reasonable, normative analysis and work environment design template. The platform establishes an integrated design and analysis system accord with our institute model development characteristics and the "five properties" quality requirements. It can make project requirements and work flow specification in our institute within a unified "five properties" job analysis. At the same time, the use of "five properties" integration platform's professional software preservation engineering data information can lay a good foundation work for new research model development, improved model's development and new employee training.

\section{References}

[1] Ma Shaomin. Integrated support engineering [M]. National Defense Industry Press, 1995.

[2] The basic concept of equipment support terms. Http://210.79.226.16: 81 1.htm /cetin2/QRMSJjczsbzx.

[3] Xu Zongchang, Huang Yijia, Yang Hongwei. Equipment support engineering and management [M]. National defense industry press, 2006:3-12.

[4] Zou Xiaojun. The integration of the military equipment maintenance mode of [D]. Changsha: National University of defense technology, 2007.

[5] Wang Hangong, Chen Xuechu, Mao Zhi Gan, etc.. Full life management of equipment system [M]. National defense industry press, 2003:198-211.

[6] LV chuan. Maintainability design and verification of [M]. National Defense Industry Press, 2012.

[7] Zhao Yu. Reliability data analysis [M]. National Defense Industry Press, 2012. 